\title{
Short Bowel Patients Treated for Two Years with Glucagon-Like Peptide 2: Effects on Intestinal Morphology and Absorption, Renal Function, Bone and Body Composition, and Muscle Function
}

\author{
P. B. Jeppesen, ${ }^{1,2}$ P. Lund, ${ }^{1}$ I. B. Gottschalck, ${ }^{1}$ H. B. Nielsen, ${ }^{3}$ J. J. Holst, ${ }^{4}$ J. Mortensen, ${ }^{5}$ \\ S.S. Poulsen, ${ }^{4}$ B. Quistorff, ${ }^{4}$ and P. B. Mortensen ${ }^{1}$ \\ ${ }^{1}$ Department of Gastroenterology CA-2121, Rigshospitalet, Blegdamsvej 9, DK-2100 Copenhagen, Denmark \\ ${ }^{2}$ Department of Medicine CA-2121, Rigshospitalet, Blegdamsvej 9, DK-2100 Copenhagen, Denmark \\ ${ }^{3}$ Department of Anesthesia, Rigshospitalet, Blegdamsvej 9, DK-2100 Copenhagen, Denmark \\ ${ }^{4}$ Department of Biomedical Sciences, The Panum Institute, Nørre Alle 20, DK-2200 Copenhagen, Denmark \\ ${ }^{5}$ Department of Clinical Physiology and Nuclear Medicin, Rigshospitalet, Blegdamsvej 9, DK-2100 Copenhagen, Denmark
}

Correspondence should be addressed to P. B. Jeppesen, bekker@dadlnet.dk

Received 24 March 2009; Revised 22 June 2009; Accepted 25 June 2009

Recommended by Karel Geboes

\begin{abstract}
Background and aims. In a short-term study, Glucagon-like peptide 2 (GLP-2) has been shown to improve intestinal absorption in short bowel syndrome (SBS) patients. This study describes longitudinal changes in relation to GLP-2 treatment for two years. Methods. GLP-2, 400 micrograms, s.c.,TID, were offered, to eleven SBS patients keeping parenteral support constant. 72-hour nutritional balance studies were performed at baseline, weeks 13, 26, 52 during two years intermitted by an 8-week washout period. In addition, mucosal morphometrics, renal function (by creatinine clearance), body composition and bone mineral density (by DEXA), biochemical markers of bone turnover (by s-CTX and osteocalcin, PTH and vitamin D), and muscle function (NMR, lungfunction, exercise test) were measured. Results. GLP-2 compliance was $>93 \%$. Three of eleven patients did not complete the study. In the remaining 8 patients, GLP-2 significantly reduced the fecal wet weight from approximately 3.0 to approximately $2.0 \mathrm{~kg} /$ day. This was accompanied by a decline in the oral wet weight intake, maintaining intestinal wet weight absorption and urinary weight constant. Renal function improved. No significant changes were demonstrated in energy intake or absorption, and GLP-2 did not significantly affect mucosal morphology, body composition, bone mineral density or muscle function. Conclusions. GLP-2 treatment reduces fecal weight by approximately $1000 \mathrm{~g} / \mathrm{d}$ and enables SBS patients to maintain their intestinal fluid and electrolyte absorption at lower oral intakes. This was accompanied by a $28 \%$ improvement in creatinine clearance.
\end{abstract}

Copyright (C) 2009 P. B. Jeppesen et al. This is an open access article distributed under the Creative Commons Attribution License, which permits unrestricted use, distribution, and reproduction in any medium, provided the original work is properly cited.

\section{Introduction}

GLP-2 is cosecreted with GLP-1 from the enteroendocrine L cells following nutrient ingestion [1]. In animal and human studies, GLP-2 decreases gastric acid secretion [2], inhibits antral gastric emptying $[3,4]$ and upregulates intestinal blood-flow [5]. In addition, GLP-2 has been demonstrated to have trophic effects on the intestinal mucosa [6-9] and positive effects on the absorptive function [10-12]. Furthermore, GLP-2 decreases bone resorption [13].
Theoretically, treatment with GLP-2 could reduce the rapid gastric emptying and hypersecretion and increase the intestinal absorption in short bowel syndrome (SBS) patients. In addition, GLP-2 could decelerate bone losses and diminish osteoporosis often described in these patients [14]. Therefore, native GLP-2 and a dipeptidyl-peptidase IV (DPP-IV) degradation-resistant gly-2 GLP-2 analog [7], Teduglutide, have been evaluated in short-term, "proof of concept" studies in the treatment of SBS patients [15, 16]. The positive effects on intestinal morphology were 
confirmed by increases in small intestinal villus heights and crypt depths, and the positive absorptive effects by increases in the wet weight, and to a minor degree, in the energy absorption. Positive effects on bone mineral content were also described [17]. However, the long-term effects of GLP-2 treatment in SBS patients remain to be evaluated.

This open-label study describes the effects of GLP-2, $400 \mathrm{mcg}$ offered for subcutaneous injection TID, to eleven patients for two consecutive years intermitted by an 8-week washout period. As a part of the study design, parenteral support was kept constant during the two years in order to evaluate longitudinal changes in the intestinal absorption and dietary intake in relation to GLP-2 treatment. Mucosal morphology, renal function, body composition, bone mineral density and muscle function were also recorded.

\section{Material and Methods}

2.1. Patients. Eleven SBS patients ( 3 female, 8 male; $47 \pm 11$ years; remnant small bowel $157 \pm 66 \mathrm{~cm}$; 2 with a colon; 7 had intestinal failure, 3 receiving parenteral fluids and electrolytes exclusively and 4 receiving parenteral nutrition; 4 had intestinal insufficiency and did not need parenteral nutrition or fluid) were recruited to the study based on a fecal energy excretion exceeding $2.0 \mathrm{MJ} / \mathrm{d}$ (measured at a previous admission) or a remnant small bowel of $200 \mathrm{~cm}$ or less (measured perioperatively from the ligament of Treitz) (Table 1 ). One patient had previously received native GLP$2(\mathrm{OBJ})$ and two teduglutide (LM and HRM) in the shortterm experiments, whereas the remaining 8 patients were GLP-2 treatment naïve. All patients, except FL and LM, took antidiarrheal medication (codein, loperamide or opium) and 6 of eleven patients (HM, OB, EFP, JE, JHJ and UDJ) took antisecretory agents (omeprazole).

2.2. Study Protocol. Over the two years, the patients were admitted at least eight times to the hospital for 72-hours evaluations (Table 2). After the baseline evaluations, the patients were given native GLP-2, 400 mcg TID, subcutaneous, for one year. For these studies we employed synthetic human GLP-2, as described previously [15]. During the first year, the patients were scheduled for readmissions at 13, 26 and 52 weeks (abbreviated Y1-W13, Y1-W26, Y1-W52, resp.,). After the first readmission at week 13, the patients were given an option to test a double dose of GLP-2, $800 \mathrm{mcg}$ TID for 3 weeks. The patients, who accepted, were readmitted for an extra 72-hour nutrient balance study at week 17 (Y1W17). After completing this evaluation, the original GLP-2 dose was reintroduced. GLP-2 treatment was discontinued for 8 weeks after the first 52 weeks of treatment. After a 72-hour washout evaluation, the GLP-2 treatment, $400 \mathrm{mcg}$ TID, was reintroduced and evaluations were repeated during admission at 13, 26 and 52 weeks during the second year of treatment (abbreviated Y2-W13, Y2-W26, Y2-W52, resp.,). In relation to the week 26 readmission, during the second year of GLP-2 treatment, the patients were given cholylsarcosine bile acid replacement therapy, 2 grams TID, two days prior to the admission and during the 72-hour balance studies $[18,19]$. Cholylsarcosine was supplied as the water soluble sodium salt, $>99 \%$ pure by HPLC and thin-layer chromatography, and was packed into gelatine capsules (250 mg/capsule) [20]. Cholylsarcosine was taken in relation to the three main meals and in conjunction with subcutaneous GLP-2 injections.

2.2.1. Morphological Analysis. At least two small bowel biopsy specimens were obtained before GLP-2 treatment at baseline and repeated at week 52, year 1, in 6 of 7 patients with a jejunostomy. Histologic sections of the biopsies were analyzed by morphometric methods (Image pro plus) as described previously [15].

2.2.2. Fluid, Electrolyte and Nutrient Balance Studies. The study-and collection-period began at 9 o'clock on the first day of admission, where patients were requested to urinate, defecate or empty their stoma-bags. During the 72-hour balance periods, all ad libitum oral intakeand stomal output were weighed, and the contents of energy (bomb calorimetry), carbohydrate (Englyst's method), nitrogen (Kjeldahl's method), fat (gas liquid chromatography), sodium and potassium (flame photometry), calcium and magnesium (atomic absorption spectrophotometry) were determined as previously described $[21,22]$. The absolute intestinal absorption was calculated as the difference between the ingested and excreted and the relative as the absolute absorption divided by oral intake.

The medication and parenteral supplements were fixed according to the status at baseline.

\subsubsection{Urine Creatinine Excretion and Creatinine Clearence.} Urinary creatinine was measured at $505 \mathrm{~nm}$ as a pikratcreatinine complex using a standard hospital analytical technique according to the method of Jaffe and the 72-hour output calculated. The creatinine clearance was calculated by diving daily urinary creatine excretion by the plasma creatinine concentration.

2.2.4. Assessment of Body Weight, Body Composition and Bone Mineral Density. The fasting body weights were measured every morning after emptying of the bladder and stomabags, before breakfast, using a leveled platform scale, and were calculated as the mean for 4 consecutive days. Body composition (BC) and bone mineral density (BMD) of the posterior-anterior spine, hip and total body were measured by Dual-energy X-ray Absorptiometry (Norland XR-36 DXA densitometer, Norland Corp., Fort Atkinson, WI., USA).

2.2.5. Biochemical Markers of Bone Turnover. Bone resorption was assessed from the concentration of s-CTX (Serum CrossLabs one step ELISA; Nordic Bioscience, Denmark) [23]. Bone formation was assessed from the concentration of s-osteocalcin (Osteocalcin N-MID ELISA assay, Nordic Bioscience, Denmark) [23].

2.2.6. Evaluation of Lung Function and Maximal Inspiratory and Expiratory Force. Lung function was tested by dynamic 


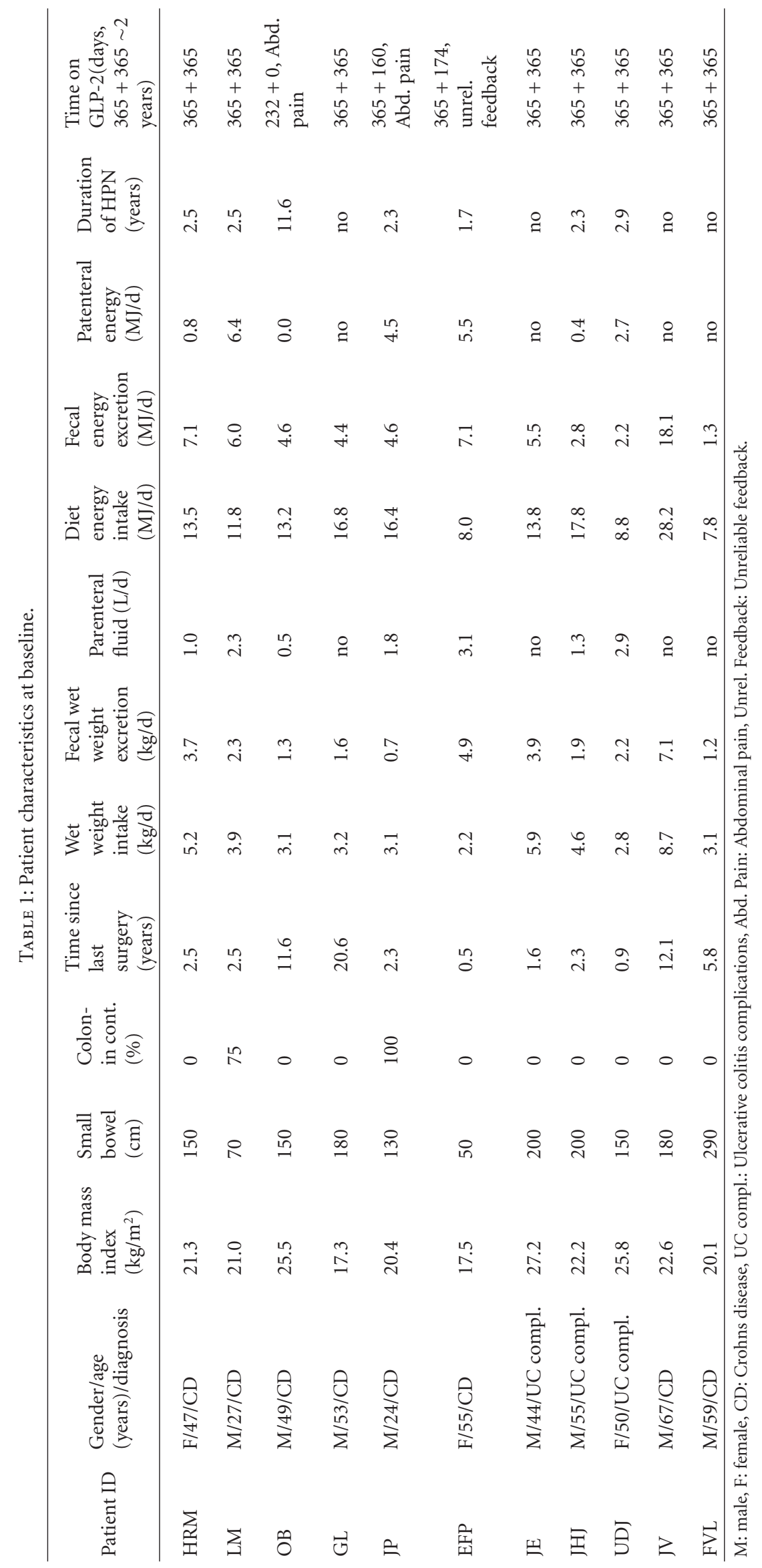


and static spirometry and measurement of single-breath diffusion capacity of carbon monoxide (MasterLab plethysmograph, Jaeger, Germany) according to the recommendations of the European Respiratory Society [24, 25]. Results were expressed in absolute values and as percentage of predicted values calculated according to European reference equations. Respiratory muscle strength was assessed by measurement of maximal expiratory and inspiratory pressures [26].

\subsubsection{Evaluation of Maximal Aerobic ATP Turnover of Skeletal} Muscle Mitochondria. The ${ }^{31} \mathrm{P}$ NMR spectroscopy investigations were conducted in a short $26 \mathrm{~cm}$ bore magnet at $2.9 \mathrm{~T}$ as described previously [27-29]. The examination of the forearm flexor muscles and the tibialis anterior muscle of the lower leg was done as two separate experiments on the same day and both protocols involved: a 5 minutes rest, three minutes of dynamic exercise at $50 \%$ of maximal voluntary contraction followed by 10 minutes of recovery. The aim of the measurements was to obtain from the $\mathrm{pH}$ and $\mathrm{PCr}$ recovery a measure of the capacity of aerobic ATP synthesis.

On a separate day, patients performed an exercise test on a modified Krogh cycling ergometer with the upper body in a $45^{\circ}$ position [30]. Exercise was maintained at 60 round per minute with an increase in workload by 50 Watt every third minute until exhaustion. Breath-by-breath measurements of pulmonary $\mathrm{O}_{2}$ consumption $\left(\mathrm{VO}_{2}\right)$ were made with an online gas analyzer (CPX/D, Medical Graphics, St. Paul, MN) and data were averaged every 30 seconds. Heart rate was recorded noninvasively [31].

2.3. Ethics. The Ethics Committee for Medical Research in Copenhagen, Denmark (KF 01-235/98) approved the protocol. Procedures followed were in accordance with the ethical standards of the Helsinki Declaration of 1975, as revised in 1983. Patients signed informed consent before entrance to the study.

2.4. Statistics. As the vast majority of data was normally distributed, the results are presented as means \pm standard deviations. The differences between admissions periods were tested with a Friedman repeated measures analysis of variance on ranks on using the SigmaStat for Windows Version 2.0 (1992-1995, Jandel Corporation, Erkrath, Germany) in patients completing the study. For comparisons of admission periods to the baseline period, the Dunnett method was used as the post hoc test. A value of $P<.05$ was considered significant.

\section{Results}

Results regarding compliance, safety, adverse events, quality of life and treatment satisfaction are presented separately [32]. In summary, all patients injected more than $93 \%$ of the prescribed GLP-2. Three of eleven patients did not complete the study. Two of these patients experienced abdominal pain, and in one patient, the investigator discontinued GLP-2 treatment, since the feedback from the patient regarding the administration of GLP-2 was lacking [32]. Abdominal pain could be a consequence of providing an intestinotrophic agent to patients with a relative stenosis, and caution should probably be taken, when prescribing GLP-2 or analogs to patients with a relative intestinal stenosis, a narrow stoma, or a history of chronic abdominal pain [32].

3.1. Morphological Analysis. Villus height increased in 4 out of 6 patients, but overall no significant changes were seen $(316 \pm 53 \mu \mathrm{m}$ versus $346 \pm 87 \mu \mathrm{m}, P=.37)$ in relation to GLP-2 treatment. Crypt depth increased in 3 patients, and decreased in 3 , but overall no change was seen $(160 \pm 63 \mu \mathrm{m}$ versus $140 \pm 24 \mu \mathrm{m}, P=.44)$.

3.1.1. Fluid, Electrolyte and Nutrient Balance Studies (Table 3). Fecal wet weight was significantly reduced by approximately one liter/d at all time intervals after the initiation of GLP2 treatment (range $629 \pm 862 \mathrm{~g} / \mathrm{d}$ to $1201 \pm 1389 \mathrm{~g} / \mathrm{d}$ ). The average reduction the first year was $811 \mathrm{~g} / \mathrm{d}$ and not different from the average reduction the second year of $1081 \mathrm{~g} / \mathrm{d}$, and no trend of a further increase in the effect on fecal weight beyond the 13th week of treatment was seen (Table 3 ). The fecal wet weight excretion reverted to baseline levels in the washout period after one year of treatment, but as for the first year the effect on fecal weight was fully regained 13 weeks after treatment was reinstituted the second year. The reduction in fecal wet weight was also seen in the two SBS patients with colon-in-continuity and numerically equaled the findings in the patients with a jejunostomy.

The reduction in fecal wet weight excretion was accompanied by a reduction in oral intake. However, in contrast to the prompt effect on fecal weight, the effect on oral intake gradually increased over the first year by an average from $449 \pm 1061 \mathrm{~g} / \mathrm{d}$ after 13 weeks of treatment to $611 \pm$ $813 \mathrm{~g} / \mathrm{d}$ after 26 weeks to finally $913 \pm 996 \mathrm{~g} / \mathrm{d}$ after 52 weeks (Y1-W52). Also in contrast to fecal excretions, oral intake was not reversed to baseline values during the eight weeks where treatment was stopped, but was only halved to an intermediate level of $453 \pm 641 \mathrm{~g} / \mathrm{d}$. However, in the second year the effect on oral intake was already back to levels of $902 \pm 919 \mathrm{~g} / \mathrm{d}$ after 13 weeks, comparable to effects it took 52 weeks of treatment to reach the first year. In the second year, no further effect was seen on oral intake beyond week 13 , and the effect leveled off at a decreased oral intake of $775 \pm 886 \mathrm{~g} / \mathrm{d}$ and $1058 \pm 1082 \mathrm{~g} / \mathrm{d}$ after 26 and 52 weeks of treatment, respectively. The decrease in fecal output resulted in a nearly comparable decrease in oral intake rendering absolute effect on intestinal absorption unaltered although intestinal absorption in percentage of oral intake increased (Table 3).

Similarly, the numerical increase in urine volume was small and did not reach statistical significance (on average $291 \mathrm{~g} / \mathrm{d}$ the first year and $238 \mathrm{~g} / \mathrm{d}$ the second year) rendering the overall urine excretion rather constant throughout the GLP-2 treatment periods in spite of the reduced oral intake. Fecal sodium excretions were reduced at all admissions (on average $53 \mathrm{mmol} / \mathrm{day}$ the first year and $58 \mathrm{mmol} / \mathrm{day}$ the second year) in relation to GLP-2 treatment and reverted to baseline levels during the washout period. Similarly, 
TABLE 2: Schedule of procedures and evaluations.

\begin{tabular}{|c|c|c|c|c|c|c|c|c|}
\hline & \multicolumn{4}{|c|}{ 1st year } & \multicolumn{4}{|c|}{ 2nd year } \\
\hline & Baseline & Week 13 & Week 26 & Week 52 & Washout (8 weeks post-treatment) & Week 13 & Week 26 & Week 52 \\
\hline Treatment & None & GLP-2 & GLP-2 & GLP-2 & None & GLP-2 & $\begin{array}{l}\text { GLP-2 + } \\
\text { Cholyl-sarcosine* }\end{array}$ & GLP-2 \\
\hline $\begin{array}{l}\text { Intestinal biopsy } \\
\text { (morphometry) }\end{array}$ & $\times$ & & & $\times$ & & & & \\
\hline $\begin{array}{l}\text { 72-h balance on } \\
\text { diet, feces, urine } \\
\text { (wet weight, na+, } \\
\mathrm{k}+, \mathrm{mg} 2+, \mathrm{ca} 2+\text {, } \\
\text { energy, fat, } \\
\text { carbohydrate, } \\
\text { protein) }\end{array}$ & $\times$ & $\times$ & $\times$ & $\times$ & $x$ & $\times$ & $x$ & $\times$ \\
\hline $\begin{array}{l}\text { 72-h Urinary } \\
\text { creatinine } \\
\text { excretion }\end{array}$ & $x$ & $x$ & $\times$ & $\times$ & $x$ & $x$ & $x$ & $x$ \\
\hline $\begin{array}{l}\text { DEXA (body } \\
\text { weight + body }\end{array}$ & & & & & & & & \\
\hline $\begin{array}{l}\text { composition }+ \\
\text { bone mineral } \\
\text { content) }\end{array}$ & $\times$ & $x$ & $\times$ & $x$ & $x$ & $x$ & $x$ & $x$ \\
\hline Vitamin D, PTH & $\times$ & $\times$ & $\times$ & $\times$ & $\times$ & $x$ & $x$ & $\times$ \\
\hline $\begin{array}{l}\text { Bone resorption } \\
\text { and formation } \\
\text { markers(s- } \\
\text { crosslabs, } \\
\text { osteocalcin) }\end{array}$ & $\times$ & $\times$ & $x$ & $\times$ & $x$ & & & \\
\hline NMR scan & $\times$ & $x$ & & & & & & \\
\hline $\begin{array}{l}\text { lung function }+ \text { in- } \\
\text { and expiratory } \\
\text { force }\end{array}$ & $\times$ & $\times$ & $\times$ & $x$ & & & & \\
\hline $\begin{array}{l}\text { exercise test on } \\
\text { bike }\end{array}$ & $x$ & $\times$ & & & & & & \\
\hline
\end{tabular}

* Cholylsarcosine was given two days prior to the admission and during the 72-hour balance studies.

urinary sodium excretions increased at most admissions (54 mmol/day the first year and $24 \mathrm{mmol} /$ day the second year). Although the decrease in fecal excretion and the increase in urinary excretion indicate that intestinal sodium absorption must have been increased, the $P$-value for this was not significant ( $P$-value of .06 from the data in Table 3 ). Treatment did not affect oral sodium intake in contrast to oral wet weight intake.

Both dietary intake and fecal excretions of potassium decreased with no significant change in absorption although urinary excretions increased during several sessions of measurements. Consistent changes in dietary intake, fecal excretion and intestinal absorption of calcium and magnesium were not seen. Urinary excretion of calcium did not increase in relation to GLP-2 treatment $(P=.08)$, whereas urinary magnesium excretion increased significantly $(P=$ .001) from a baseline of $2 \pm 2 \mathrm{mmol} / \mathrm{d}$ to values ranging from $3 \pm 3 \mathrm{mmol} / \mathrm{d}$ to $5 \pm 3 \mathrm{mmol} / \mathrm{d}$.

The results on the energy and macronutrient balances are presented in Table 4. On an average, treatment with GLP-2 numerically decreased oral energy intake by $349 \mathrm{~kJ} / \mathrm{d}$ in the first year and $1182 \mathrm{~kJ} / \mathrm{d}$ in the second year, which was less than $3 \%$ and $8 \%$ of baseline intake and not significant $(P=$ .69 , Table 4$)$. Fecal excretion of energy numerically decreased on an average by $448 \mathrm{~kJ} / \mathrm{d}$ in the first year and $1438 \mathrm{~kJ} / \mathrm{d}$ in the second year. None of these changes were significant. However, the combination of GLP-2 and cholylsarcosine appeared successful in reducing fecal excretions by a total of $1852 \mathrm{~kJ} / \mathrm{d}(\sim 31 \%)$, which partly appeared to be caused by a cholylsarcosine associated $42 \%$ decrease in fecal fat excretion of $28 \mathrm{~g} / \mathrm{d}(1094 \mathrm{~kJ} / \mathrm{d}, P=.005)$, as GLP-2 alone did not influence fecal fat (Table 4). Nevertheless, the accompanying reduction in dietary intake reduced the overall gain in energy absorption to a negligible amount of less than $100 \mathrm{~kJ} / \mathrm{d}$ except for the period where cholylsarcosine was added, which resulted in a numerical increase in absorption of $701 \mathrm{~kJ} /$ day or $8 \%$ of baseline, but still not enough to reach significance.

Fecal excretions of carbohydrate and protein (nitrogen) did not decrease $(P=.08)$ with no demonstrable increase in intestinal absorption (Table 4). 
TABLe 3: Diet, feces, absorption (diet-feces), and urine excretion of wet weight, sodium, potassium, calcium, and magnesium.

\begin{tabular}{|c|c|c|c|c|c|c|c|c|c|}
\hline \multirow[t]{2}{*}{$($ mean $\pm \mathrm{SD}, n=8)$} & \multirow[b]{2}{*}{ Baseline } & \multicolumn{3}{|c|}{ 1st year } & \multicolumn{4}{|c|}{ 2nd year } & \multirow[b]{2}{*}{$P$-value* } \\
\hline & & Week 13 & Week 26 & Week 52 & Washout & Week 13 & Week 26 & Week 52 & \\
\hline Treatment & None & GLP-2 & GLP-2 & GLP-2 & None & GLP-2 & $\begin{array}{l}\text { GLP-2 + } \\
\text { Cholyl- } \\
\text { sarcosine\# }\end{array}$ & GLP-2 & \\
\hline \multicolumn{10}{|l|}{ Wet weight (g/d) } \\
\hline Diet & $4680 \pm 1945$ & $4232 \pm 1813 \S$ & $4069 \pm 1523 \S$ & $3768 \pm 1355 \S$ & $4228 \pm 1589 \S$ & $3779 \pm 1254 \S$ & $\begin{array}{l}3905 \pm \\
1293 \S\end{array}$ & $3623 \pm 1047 \S$ & .03 \\
\hline Feces & $2995 \pm 1905$ & $2136 \pm 1388 \S$ & $2366 \pm 391 \S$ & $2048 \pm 1158 \S$ & $2759 \pm 1371$ & $1853 \pm 1093 \S$ & $\begin{array}{l}2094 \pm \\
1139 \S\end{array}$ & $1794 \pm 771 \S$ & $<.001$ \\
\hline Abs. absorption & $1686 \pm 578$ & $2096 \pm 600$ & $1702 \pm 784$ & $1720 \pm 506$ & $1468 \pm 627$ & $1926 \pm 854$ & $1811 \pm 679$ & $1929 \pm 656$ & .11 \\
\hline Rel. absorption & $39 \pm 16$ & $52 \pm 13 \S$ & $41 \pm 16$ & $48 \pm 14$ & $35 \pm 17$ & $52 \pm 18 \S$ & $47 \pm 18$ & $50 \pm 15$ & $<.001$ \\
\hline Urine & $1407 \pm 860$ & $1740 \pm 756$ & $1720 \pm 749$ & $1635 \pm 774$ & $1410 \pm 807$ & $1855 \pm 1002$ & $1528 \pm 917$ & $1551 \pm 596$ & .16 \\
\hline \multicolumn{10}{|l|}{ Sodium $(\mathrm{mmol} / \mathrm{d})$} \\
\hline Diet & $238 \pm 116$ & $233 \pm 126$ & $228 \pm 117$ & $209 \pm 118$ & $223 \pm 109$ & $222 \pm 113$ & $203 \pm 101$ & $216 \pm 104$ & .41 \\
\hline Feces & $264 \pm 132$ & $197 \pm 113 \S$ & $234 \pm 96 \S$ & $203 \pm 96 \S$ & $250 \pm 150$ & $198 \pm 108 \S$ & $226 \pm 119 \S$ & $195 \pm 79 \S$ & .001 \\
\hline Abs. absorption & $-26 \pm 84$ & $36 \pm 64$ & $-6 \pm 89$ & $6 \pm 71$ & $-27 \pm 126$ & $25 \pm 74$ & $-23 \pm 96$ & $21 \pm 61$ & .06 \\
\hline Rel. absorption & $-24 \pm 72$ & $13 \pm 29$ & $-25 \pm 81$ & $-18 \pm 74$ & $-27 \pm 78$ & $-8 \pm 92$ & $-32 \pm 95$ & $-2 \pm 55$ & .15 \\
\hline Urine & $77 \pm 139$ & $139 \pm 80 \S$ & $133 \pm 50 \S$ & $122 \pm 55 \S$ & $59 \pm 72$ & $124 \pm 64 \S$ & $78 \pm 61$ & $100 \pm 66$ & .001 \\
\hline \multicolumn{10}{|l|}{ Potassium (mmol/d) } \\
\hline Diet & $117 \pm 45$ & $107 \pm 43 \S$ & $111 \pm 38 \S$ & $100 \pm 43 \S$ & $104 \pm 43 \S$ & $93 \pm 34 \S$ & $90 \pm 26 \S$ & $90 \pm 24 \S$ & .007 \\
\hline Feces & $49 \pm 41$ & $32 \pm 31 \S$ & $31 \pm 28 \S$ & $28 \pm 24 \S$ & $46 \pm 30$ & $24 \pm 21 \S$ & $26 \pm 23 \S$ & $23 \pm 22 \S$ & $<.001$ \\
\hline Abs. absorption & $68 \pm 43$ & $76 \pm 37$ & $80 \pm 28$ & $73 \pm 36$ & $58 \pm 38$ & $70 \pm 36$ & $64 \pm 30$ & $67 \pm 26$ & .21 \\
\hline Rel. absorption & $58 \pm 24$ & $72 \pm 28$ & $74 \pm 19 \S$ & $72 \pm 24$ & $56 \pm 30$ & $73 \pm 28$ & $72 \pm 25$ & $75 \pm 22 \S$ & $<.001$ \\
\hline Urine & $62 \pm 16$ & $73 \pm 13$ & $86 \pm 25 \S$ & $73 \pm 19$ & $49 \pm 16$ & $87 \pm 19 \S$ & $64 \pm 24$ & $86 \pm 20 \S$ & $<.001$ \\
\hline \multicolumn{10}{|l|}{ Calcium (mmol/d) } \\
\hline Diet & $49 \pm 30$ & $44 \pm 27$ & $42 \pm 20$ & $34 \pm 20 \S$ & $39 \pm 28 \S$ & $32 \pm 19 \S$ & $31 \pm 18 \S$ & $32 \pm 20 \S$ & $<.001$ \\
\hline Feces & $66 \pm 52$ & $65 \pm 42$ & $70 \pm 41$ & $61 \pm 42$ & $63 \pm 43$ & $50 \pm 31 \S$ & $49 \pm 32 \S$ & $45 \pm 24 \S$ & $<.001$ \\
\hline Abs. absorption & $-17 \pm 35$ & $-21 \pm 26$ & $-28 \pm 27 \S$ & $-27 \pm 28 \S$ & $-25 \pm 25 \S$ & $-18 \pm 20$ & $-18 \pm 26$ & $-13 \pm 20$ & .009 \\
\hline Rel. absorption & $-39 \pm 59$ & $-55 \pm 60$ & $-72 \pm 63$ & $-89 \pm 82$ & $-87 \pm 76$ & $-69 \pm 60$ & $-77 \pm 112$ & $-66 \pm 85$ & .24 \\
\hline Urine & $3 \pm 3$ & $3 \pm 3$ & $4 \pm 4$ & $4 \pm 4$ & $4 \pm 4$ & $5 \pm 4$ & $5 \pm 4$ & $4 \pm 3$ & .08 \\
\hline \multicolumn{10}{|c|}{ Magnesium (mmol/d) } \\
\hline Diet & $19 \pm 8$ & $18 \pm 8$ & $19 \pm 7$ & $17 \pm 7 \S$ & $18 \pm 7$ & $16 \pm 6 \S$ & $16 \pm 5 \S$ & $15 \pm 4 \S$ & .03 \\
\hline Feces & $32 \pm 27$ & $31 \pm 32$ & $34 \pm 33$ & $33 \pm 35$ & $34 \pm 30$ & $29 \pm 27$ & $28 \pm 25$ & $30 \pm 29$ & .01 \\
\hline Abs. absorption & $-12 \pm 21$ & $-12 \pm 25$ & $-15 \pm 27$ & $-16 \pm 28$ & $-16 \pm 25$ & $-13 \pm 23$ & $-12 \pm 22$ & $-15 \pm 26$ & .10 \\
\hline Rel. absorption & $-51 \pm 80$ & $-42 \pm 89$ & $-60 \pm 92$ & $-67 \pm 99$ & $-73 \pm 105$ & $-61 \pm 108$ & $-60 \pm 119$ & $-79 \pm 128$ & .17 \\
\hline Urine & $2 \pm 2$ & $3 \pm 3 \S$ & $4 \pm 3 \S$ & $4 \pm 4 \S$ & $3 \pm 3 \S$ & $5 \pm 3 \S$ & $4 \pm 2 \S$ & $4 \pm 2 \S$ & .001 \\
\hline
\end{tabular}

*Friedman repeated measures analysis of variance on ranks in patients completing the study. For comparisons of admission periods to the baseline period, the Dunnett method was used as the post hoc test. $\S \sim P<.05$. \#Cholylsarcosine was given two days prior to the admission and during the 72 -hour balance studies.

Six of the eight patients completing the study opted to try the double dose of GLP-2, 800 mcg TID for 3 weeks and were readmitted for an extra 72-hour nutrient balance study (Y1W17, Patients HRM, LM, GL, JE, JHJ and JV). Compared to effects at week 13, doubling the GLP-2 dose for three weeks did not significantly affect wet weight intake $(4761 \pm 1780 \mathrm{~g} / \mathrm{d}$ versus $4561 \pm 1730 \mathrm{~g} / \mathrm{d}, P=.84$ ), fecal wet weight excretion $(2469 \pm 1468 \mathrm{~g} / \mathrm{d}$ versus $2484 \pm 1678 \mathrm{~g} / \mathrm{d}, P=1.00)$ or urine wet weight excretion $(1634 \pm 857 \mathrm{~g} / \mathrm{d}$ versus $1752 \pm 848 \mathrm{~g} / \mathrm{d}$, $P=.18$ ). Sodium, potassium, calcium and magnesium balances were unaffected by the doubling of the GLP-2 dose. Likewise, compared to effects at week 13, doubling the GLP2 dose for three weeks did not significantly affect diet energy intake $(16123 \pm 5373 \mathrm{~kJ} / \mathrm{d}$ versus $16178 \pm 6354, P=.56)$, fecal energy excretion $(6646 \pm 3817 \mathrm{~kJ} / \mathrm{d}$ versus $6657 \pm 4745 \mathrm{~kJ} / \mathrm{d}$, $P=1.00)$ or carbohydrate, fat or protein balances.
3.1.2. Urine Creatinine Excretion and Creatinine Clearance (Table 5). On an average GLP-2 increased creatinine clearance by $20 \mathrm{~mL} / \mathrm{min}$ or $28 \%$ in both years of treatment $(P=.04)$ from a baseline value of $73 \pm 22 \mathrm{~mL} / \mathrm{min}$ (normal range $48-150 \mathrm{~mL} / \mathrm{min}$ ) to a range between $91 \pm 14 \mathrm{~mL} / \mathrm{min}$ and $97 \pm 21 \mathrm{~mL} / \mathrm{min}$ during the first year and a range between $85 \pm 36 \mathrm{~mL} / \mathrm{min}$ and $105 \pm 23 \mathrm{~mL} / \mathrm{min}$ during the second year. Creatinine clearence reverted to baseline levels $(67 \pm 43 \mathrm{~mL} / \mathrm{min})$ during the washout period. Corresponding changes were seen in plasma and urine creatinine.

3.2. Assessment of Body Weight and Composition, Bone Mineral Density, Biochemical Markers of Bone Turnover (SCrosslabs and S-Osteocalcin), Vitamin D and PTH (Table 6). Although the body weight numerically increased from $69.2 \pm$ 9.1 at baseline to $72.6 \pm 13.7$ after two years of GLP-2 
TABLE 4: Diet, feces, and absorption (diet-feces) of energy, fat, carbohydrate, and protein.

\begin{tabular}{|c|c|c|c|c|c|c|c|c|c|}
\hline \multirow[t]{2}{*}{$($ mean $\pm \mathrm{SD}, n=8)$} & \multirow[b]{2}{*}{ Baseline } & \multicolumn{2}{|c|}{ 1st year } & \multicolumn{6}{|c|}{ 2nd year } \\
\hline & & Week 13 & Week 26 & Week 52 & Washout & Week 13 & Week 26 & Week 52 & $P$-value* \\
\hline Treatment & None & GLP-2 & GLP-2 & GLP-2 & None & GLP-2 & $\begin{array}{l}\text { GLP-2 + } \\
\text { Cholyl- } \\
\text { sarcosine\# }\end{array}$ & GLP-2 & \\
\hline \multicolumn{10}{|l|}{ Energy $(\mathrm{kJ} / \mathrm{d})$} \\
\hline Diet & $14810 \pm 6429$ & $14421 \pm 5556$ & $14958 \pm 5485$ & $14001 \pm 5445$ & $15277 \pm 5926$ & $13729 \pm 5369$ & $\begin{array}{l}13658 \pm \\
4821\end{array}$ & $13494 \pm 4025$ & .69 \\
\hline Feces & $5928 \pm 5305$ & $5400 \pm 3968$ & $5799 \pm 4095$ & $5240 \pm 3668$ & $6332 \pm 4197$ & $4838 \pm 3469$ & $4075 \pm 2866$ & $4555 \pm 2596$ & .02 \\
\hline Abs. absorption & $8882 \pm 3339$ & $9021 \pm 2500$ & $9159 \pm 2151$ & $8761 \pm 2461$ & $8945 \pm 2655$ & $8892 \pm 2786$ & $9583 \pm 2649$ & $8940 \pm 2419$ & .82 \\
\hline Rel. absorption & $64 \pm 18$ & $66 \pm 16$ & $64 \pm 13$ & $65 \pm 12$ & $61 \pm 16$ & $66 \pm 15$ & $72 \pm 12$ & $67 \pm 14$ & .03 \\
\hline \multicolumn{10}{|l|}{ Fat $(\mathrm{g} / \mathrm{d})$} \\
\hline Diet & $103 \pm 43$ & $113 \pm 44$ & $114 \pm 46$ & $103 \pm 40$ & $109 \pm 46$ & $104 \pm 44$ & $98 \pm 45$ & $103 \pm 42$ & .22 \\
\hline Feces & $67 \pm 62$ & $69 \pm 61$ & $70 \pm 58$ & $61 \pm 58$ & $78 \pm 63$ & $58 \pm 56$ & $39 \pm 40 \S$ & $54 \pm 47$ & .005 \\
\hline Abs. absorption & $36 \pm 35$ & $44 \pm 25$ & $44 \pm 20$ & $42 \pm 33$ & $31 \pm 29$ & $46 \pm 28$ & $59 \pm 26$ & $48 \pm 24$ & .11 \\
\hline Rel. absorption & $43 \pm 34$ & $48 \pm 36$ & $46 \pm 29$ & $50 \pm 37$ & $35 \pm 36$ & $50 \pm 33$ & $64 \pm 27 \S$ & $50 \pm 34$ & .002 \\
\hline \multicolumn{10}{|l|}{ Carbohydrate (g/d) } \\
\hline Diet & $374 \pm 145$ & $345 \pm 128$ & $354 \pm 129$ & $341 \pm 126$ & $382 \pm 140$ & $342 \pm 129$ & $355 \pm 107$ & $369 \pm 102$ & .62 \\
\hline Feces & $66 \pm 60$ & $57 \pm 43$ & $59 \pm 40$ & $57 \pm 37$ & $70 \pm 42$ & $52 \pm 33$ & $50 \pm 31$ & $51 \pm 19$ & .08 \\
\hline Abs. absorption & $308 \pm 109$ & $288 \pm 97$ & $295 \pm 100$ & $284 \pm 96$ & $311 \pm 106$ & $290 \pm 107$ & $306 \pm 81$ & $318 \pm 90$ & .53 \\
\hline Rel. absorption & $84 \pm 9$ & $84 \pm 6$ & $84 \pm 5$ & $84 \pm 5$ & $83 \pm 6$ & $85 \pm 5$ & $87 \pm 4$ & $86 \pm 3$ & .18 \\
\hline \multicolumn{10}{|l|}{ Protein $(\mathrm{g} / \mathrm{d})$} \\
\hline Diet & $105 \pm 51$ & $101 \pm 48$ & $107 \pm 44$ & $96 \pm 52$ & $115 \pm 54$ & $99 \pm 41$ & $95 \pm 39$ & $102 \pm 41$ & .15 \\
\hline Feces & $50 \pm 35$ & $46 \pm 31$ & $47 \pm 27$ & $46 \pm 29$ & $57 \pm 35$ & $43 \pm 34$ & $42 \pm 22$ & $43 \pm 22$ & .08 \\
\hline Abs. absorption & $55 \pm 36$ & $54 \pm 31$ & $60 \pm 25$ & $49 \pm 33$ & $58 \pm 33$ & $56 \pm 29$ & $53 \pm 33$ & $59 \pm 32$ & .76 \\
\hline Rel. absorption & $52 \pm 22$ & $55 \pm 19$ & $57 \pm 13$ & $50 \pm 18$ & $51 \pm 21$ & $55 \pm 19$ & $54 \pm 18$ & $56 \pm 18$ & .50 \\
\hline
\end{tabular}

${ }^{*}$ Friedman repeated measures analysis of variance on ranks in patients completing the study. For comparisons of admission periods to the baseline period, the Dunnett method was used as the post hoc test. $\S \sim P<.05$. \# Cholylsarcosine was given two days prior to the admission and during the 72-hour balance studies.

TABLE 5: Plasma creatinine, urine creatinine, and creatinine clearence.

\begin{tabular}{|c|c|c|c|c|c|c|c|c|c|}
\hline \multirow{2}{*}{$\begin{array}{l}\text { (mean } \\
\mathrm{SD}, n=8)\end{array}$} & \multirow[b]{2}{*}{ Baseline } & \multicolumn{3}{|c|}{ 1st year } & \multicolumn{4}{|c|}{ 2nd year } & \multirow[b]{2}{*}{$P$-value* } \\
\hline & & Week 13 & Week 26 & Week 52 & Washout & Week 13 & Week 26 & Week 52 & \\
\hline Treatment & None & GLP-2 & GLP-2 & GLP-2 & None & GLP-2 & $\begin{array}{l}\text { GLP-2 + } \\
\text { Cholyl- } \\
\text { sarcosine\# }\end{array}$ & GLP-2 & \\
\hline $\begin{array}{l}\text { Plasma } \\
\text { Creatinine } \\
(\mathrm{mmol} / \mathrm{l})\end{array}$ & $0.103 \pm 0.019$ & $0.097 \pm 0.019 \S$ & $0.096 \pm 0.019 \S$ & $0.095 \pm 0.020 \S$ & $0.094 \pm 0.023 \S$ & $0.088 \pm 0.018 \S$ & $\begin{array}{l}0.101 \pm \\
0.029 \S\end{array}$ & $0.086 \pm 0.024 \S$ & .01 \\
\hline $\begin{array}{l}\text { Urine } \\
\text { Creatinine } \\
(\mathrm{mmol} / \mathrm{d})\end{array}$ & $11 \pm 3$ & $13 \pm 4$ & $13 \pm 4$ & $13 \pm 4$ & $8 \pm 4$ & $11 \pm 3$ & $12 \pm 5$ & $13 \pm 4$ & .016 \\
\hline $\begin{array}{l}\text { Creatinine } \\
\text { Clearence } \\
(\mathrm{mL} / \mathrm{min})\end{array}$ & $73 \pm 22$ & $91 \pm 14 \S$ & $97 \pm 21 \S$ & $93 \pm 28 \S$ & $67 \pm 43$ & $90 \pm 26 \S$ & $85 \pm 36 \S$ & $105 \pm 23 \S$ & .04 \\
\hline
\end{tabular}

treatment, this did not reach statistical significance $(P=.25)$. No significant changes occurred regarding lean body mass and fat mass, and bone mineral content was constant in relation to the two years of GLP-2 treatment.

Significant, but sporadic, differences between admission periods were demonstrated regarding bone mineral density of the femoral neck and the distal radius and ulna, but the post hoc analyses were nonsignificant. No significant changes were seen regarding $\mathrm{z}$-scores of the femoral neck or the spine.
S-crosslabs decreased significantly at week 13 . Vitamin D fluctuations were probably related to the season of the year. Osteocalcin and PTH remained constant throughout the two years of GLP-2 treatment.

3.3. Evaluation of Physical Strength and Mitochondrial Function of Skeletal Muscle (Lung Function and Maximal Inspiratory and Expiratory Force, NMR Turnover Test and Exercise Test). Measurements of lung function at baseline 
TABLE 6: Body weight and composition, bone mineral density, biochemical markers of bone turnover (s-crosslabs and s-osteocalcin), vitamin $\mathrm{D}$ and PTH.

\begin{tabular}{|c|c|c|c|c|c|c|c|c|c|}
\hline \multirow[t]{2}{*}{$($ mean $\pm \mathrm{SD})$} & \multirow[b]{2}{*}{ Baseline } & \multicolumn{2}{|c|}{ 1st year } & \multicolumn{6}{|c|}{ 2nd year } \\
\hline & & Week 13 & Week 26 & Week 52 & Washout & Week 13 & Week 26 & Week 52 & $P$-value* \\
\hline Treatment & None & GLP-2 & GLP-2 & GLP-2 & None & GLP-2 & $\begin{array}{l}\text { GLP-2+ } \\
\text { Cholyl- } \\
\text { sarcosine\# }\end{array}$ & GLP-2 & \\
\hline \multicolumn{10}{|l|}{ Body composition, kg } \\
\hline Body weight & $69.2 \pm 9.1$ & $70.8 \pm 9.5$ & $71.2 \pm 9.7$ & $70.2 \pm 12.3$ & $70.2 \pm 14.0$ & $71.8 \pm 12.9$ & $71.3 \pm 12.3$ & $72.6 \pm 13.7$ & .25 \\
\hline Lean body mass & $45.7 \pm 9.5$ & $47.3 \pm 9.3$ & $47.4 \pm 9.9$ & $48.0 \pm 10.0$ & $46.7 \pm 9.6$ & $48.0 \pm 10.0$ & $47.1 \pm 9.9$ & $46.9 \pm 10.3$ & .46 \\
\hline Fat mass & $20.1 \pm 8.9$ & $19.4 \pm 8.8$ & $20.2 \pm 9.0$ & $19.3 \pm 8.8$ & $20.0 \pm 8.2$ & $20.7 \pm 8.9$ & $20.7 \pm 8.6$ & $21.8 \pm 8.7$ & .07 \\
\hline Bone mineral content & $2.67 \pm 0.27$ & $2.71 \pm 0.32$ & $2.71 \pm 0.29$ & $2.73 \pm 0.30$ & $2.70 \pm 0.29$ & $2.74 \pm 0.29$ & $2.74 \pm 0.29$ & $2.73 \pm 0.26$ & .75 \\
\hline \multicolumn{10}{|c|}{ Bone mineral density, $\mathrm{g} / \mathrm{cm}^{2}$} \\
\hline Spine & $0.97 \pm 0.25$ & $0.96 \pm 0.26$ & $0.97 \pm 0.26$ & $0.95 \pm 0.24$ & $0.95 \pm 0.24$ & $0.95 \pm 0.22$ & $0.96 \pm 0.23$ & $0.96 \pm 0.22$ & .49 \\
\hline Femoral neck & $0.79 \pm 0.14$ & $0.80 \pm 0.16$ & $0.79 \pm 0.14$ & $0.78 \pm 0.14$ & $0.78 \pm 0.13$ & $0.76 \pm 0.13$ & $0.78 \pm 0.12$ & $0.77 \pm 0.14$ & .04 \\
\hline Dist. Rad+Uln & $0.37 \pm 0.05$ & $0.37 \pm 0.05$ & $0.36 \pm 0.05$ & $0.37 \pm 0.05$ & $0.37 \pm 0.05$ & $0.37 \pm 0.05$ & $0.38 \pm 0.05$ & $0.37 \pm 0.05$ & .02 \\
\hline Prox. Rad+Uln & $0.77 \pm 0.06$ & $0.77 \pm 0.07$ & $0.77 \pm 0.06$ & $0.78 \pm 0.06$ & $0.78 \pm 0.07$ & $0.78 \pm 0.06$ & $0.78 \pm 0.06$ & $0.78 \pm 0.07$ & .27 \\
\hline Prox. Rad & $0.77 \pm 0.07$ & $0.77 \pm 0.06$ & $0.76 \pm 0.06$ & $0.77 \pm 0.05$ & $0.77 \pm 0.06$ & $0.77 \pm 0.06$ & $0.78 \pm 0.06$ & $0.78 \pm 0.07$ & .06 \\
\hline Spine, Z-Score & $-0.70 \pm 1.78$ & $-0.80 \pm 1.86$ & $-0.71 \pm 1.89$ & $-0.57 \pm 1.83$ & $-0.82 \pm 1.81$ & $-0.82 \pm 1.67$ & $-0.83 \pm 1.70$ & $-0.71 \pm 1.68$ & .37 \\
\hline Fem. Neck, Z-Score & $-0.85 \pm 1.10$ & $-0.73 \pm 1.19$ & $-0.84 \pm 1.04$ & $-0.86 \pm 1.04$ & $-0.85 \pm 1.11$ & $-1.00 \pm 0.96$ & $-0.85 \pm 0.94$ & $-0.94 \pm 1.07$ & .23 \\
\hline S-Crosslabs, ng/mL & $0.44 \pm 0.19$ & $0.25 \pm 0.11 \S$ & $0.32 \pm 0.14$ & $0.40 \pm 0.24$ & $0.49 \pm 0.32$ & $\%$ & $\%$ & $\%$ & .04 \\
\hline S-Osteocalcin, ng/L & $24 \pm 12$ & $24 \pm 10$ & $21 \pm 9$ & $25 \pm 15$ & $24 \pm 16$ & $\%$ & $\%$ & $\%$ & .75 \\
\hline P-PTH, pmol/L & $4.5 \pm 3.2$ & $4.7 \pm 2.8$ & $4.3 \pm 2.0$ & $5.0 \pm 2.4$ & $3.8 \pm 1.7$ & $4.4 \pm 2.3$ & $5.1 \pm 3.4$ & $4.9 \pm 2.2$ & .92 \\
\hline P-Vitamin-D, nmol/L & $79 \pm 28$ & $57 \pm 25$ & $55 \pm 31$ & $91 \pm 49$ & $78 \pm 37$ & $87 \pm 52$ & $89 \pm 63$ & $92 \pm 44$ & .02 \\
\hline
\end{tabular}

*Friedman repeated measures analysis of variance on ranks in patients completing the study. For comparisons of admission periods to the baseline period, the Dunnett method was used as the post hoc test. $\S \sim P<.05$. \#Cholylsarcosine was given two days prior to the admission and during the 72-hour balance studies.

showed that 7 of $8(88 \%)$ patients had an obstructive ventilatory defect. Five of $8(63 \%)$ patients had a restrictive ventilatory defect and 8 of $8(100 \%)$ patients had decreased diffusion capacity. No change was observed in relation to the GLP-2 treatment in flow indices as for example, FEV1, static volumes as total lung capacity, residual volume and vital capacity or diffusion capacity for carbon monoxide. Yet, functional residual capacity decreased significantly from $144 \pm 23 \%$ at baseline to $128 \pm 28 \%(P<.001)$ at week 52 . Respiratory muscle strength was within the normal limits and did not change in relation to the GLP-2 treatment.

The intension of the ${ }^{31} \mathrm{P}$ NMR spectroscopic experiments was to test, if the capacity of aerobic ATP synthesis of the skeletal muscle was changed by the GLP-2 treatment. The measurements were performed in two muscle groups, the tibialis anterior, which is primarily a red muscle (type 1) [29] and the forearm flexor muscle, which is primarily of type 2 [33]. Table 7 shows the results at baseline before treatment was initiated. The differences observed between the two muscles are similar to what has been observed previously [29]. The data from the subsequent measurements at week 13 is not shown, since no significant changes were observed in any of the parameters recorded.

In relation to the exercise test, the resting heart rate and $\mathrm{VO}_{2}$ were $78 \pm 4 \mathrm{bpm}$ and $0.3 \pm 0.05 \mathrm{~L} / \mathrm{min}$, at baseline, respectively, and when patients were exhausted during cycling the heart rate and $\mathrm{VO}_{2}$ increased to $161 \pm 5 \mathrm{bpm}$ and $1.8 \pm 0.9 \mathrm{~L} / \mathrm{min}$, respectively, $(P<.05)$. Thirteen weeks of treatment with GLP-2 did not change resting and exercise heart rate and $\mathrm{VO}_{2}$.

\section{Discussion}

In contrast to the previous short-term studies, where active efforts were made to enforce strict compliance to injections and adhesion to fixed diets in hospital-like settings, this study aimed at describing the consequences of introducing and expanding long-term GLP-2 treatment to the daily life of the short bowel patients. However, parenteral support was deliberately maintained constant. GLP-2 was well tolerated as demonstrated by the high compliance in the SBS patients completing this study $(\geq 94 \%)$ but also in the patients discontinuing GLP-2 treatment ( $\geq 93 \%)$.

The effect of long-term GLP-2 treatment on intestinal morphology was evaluated in biopsies taken at baseline and after a year of GLP-2 treatment in six patients with a jejunostomy. No significant changes in the villus height $(10 \pm 25 \%, P=.37)$ or the crypt depth $(-5 \pm 28 \%, P=.44)$ were observed. This was in accordance with the findings in the initial 35 days GLP-2 study [15], where $400 \mu \mathrm{g}$ of native GLP-2 was given subcutaneously twice daily (corresponding to $0.013 \pm 0.002 \mathrm{mg} / \mathrm{kg} / \mathrm{d}$, a range of $0.011-0.017 \mathrm{mg} / \mathrm{kg} / \mathrm{d}$ ). No changes were seen in villus height $(10 \pm 19 \%, P=.14)$ or the crypt depth $(18 \pm 42 \%, P=0.28)$. In contrast, 21 days of teduglutide in doses ranging from 0.03 to 0.10 $\mathrm{mg} / \mathrm{kg} / \mathrm{d}[16]$ increased villus heights by $38 \pm 45 \%(P=.030)$ and crypt depth increased $22 \pm 18 \%(P=.010)$ in eight short bowel jejunostomy patients. The more pronounced positive effects of teduglutide may be due to the prolonged half-life of the peptide and subsequently by the larger area under the curve, or simply be related to the larger doses 
TABLE 7: ${ }^{31}$ PMRS measurements on primarily red (tibialis anterior) or white (forearm) skeletal muscle of subjects before treatment with GLP2. Tibialis anterior represents a primarily "red" and the forearm flexor group a primarily "white" muscle. The first 4 lines are recorded at rest where $\mathrm{PCr}$ is given as $\mathrm{mM}$. The subsequent 4 lines are recorded after a $1 \mathrm{~min}$ of maximal contraction. The $\mathrm{PCr}$ and $\mathrm{pH}$ change is the difference between the resting value and the value measured by the end of exercise. The $\mathrm{T}_{0.5}$ values are half times for recovery ( $\mathrm{s}$ ), assuming monoexponential recovery after exercise (27).

\begin{tabular}{lcr}
\hline & Forearm Flexors & Tibialis Anterior \\
\hline At rest: & & $23.5 \pm 2.3$ \\
PCr & $24.3 \pm 1.7$ & $4.3 \pm 0.4$ \\
PCr/ATP & $4.2 \pm 0.5$ & $6.0 \pm 1.5^{*}$ \\
PCr/Pi & $7.7 \pm 1.9$ & $6.99 \pm 0.05^{* *}$ \\
pH & $7.04 \pm 0.04$ & $13.7 \pm 5.7$ \\
After exercise: & & $0.18 \pm 0.17^{*}$ \\
PCr change & $14.0 \pm 3.3$ & $45.1 \pm 11.0$ \\
pH change & $0.46 \pm 0.29$ & $26.7 \pm 7.0^{+}$ \\
$T_{-}{ }^{+} 0.5 P C r "$ & $59.6 \pm 16.0$ & $0.27 \pm 0.19$ \\
$T_{-}{ }^{\prime} 0.5 P i "$ & $34.8 \pm 12.0^{+}$ & \\
$V_{-}{ }^{+}$max" & $0.25 \pm 0.08$ &
\end{tabular}

used in that study. The finding of continuously elevated endogenous GLP-2 levels and increased mucosal growth in short bowel patients with colon-in continuity and in obese patients subjected to jejunoileal bypass operations supports the "area under the curve" theory. In these patients, the endogenous meal-stimulated peak-concentrations of GLP-2 are much lower than the pharmacologically induced peaks, but a continuous endogenous GLP-2 secretion occurs, even after a night of fasting. Thus, the increases in villus height and crypt depths in short bowel patients with a jejunostomy treated with either native GLP-2 or teduglutide is still significantly less than the $80 \%$ increases in villus height demonstrated in patients with jejunoileal bypass operations [34] and the $2-300 \%$ increases in villus heights described in patients with enteroglucagonomas $[35,36]$. However, it is important to notice that local concentrations of GLP2 delivered by paracrine secretions may not reflect plasma levels.

The magnitude of the effects of GLP-2 and teduglutide on bowel morphology is important in the discussion of the potential for cancerous growth attributable to these peptides. GLP-2, and to a greater degree, teduglutide, have been shown to promote an increase in the growth of mucosal neoplasms in mice developing colonic tumors in response to the methylating carcinogen 1,2-dimethylhydralazine [37]. Despite the fact that the small bowel is the longest region of the alimentary tract, neoplasms of the small bowel are very uncommon. Therefore, in our practice, screening for small bowel neoplasms is not considered prior to initiation of GLP2 treatment or during treatment. On the other hand, cancer of the colon and rectum is the fourth most common newly diagnosed cancer in The United States and Europe. The colorectal adenomas that may predispose to this condition are even more frequent. It could be argued, that screening prior to the initiation of GLP-2 treatment would be appropriate in short bowel patients with a preserved colon. However, as stated, the patients, who have undergone jejono-ileal bypass operations, have continuously elevated endogenous GLP2 plasma concentrations. In these patients, the changes in the functional remnant jejunum clearly supersede changes obtained by pharmacological intervention, and the colonic crypts are also more enlarged. Thus, it is suggested, that there is no indication for regular colonoscopic surveillance. Although it will probably take another 10-20 years to establish whether jejuno-ileal bypass is associated with an increased risk of large bowel cancer in humans, the findings, so far, are negative [38]. The colon was normal before and after GLP-2 treatment in the two SBS patients with colon-in continuity in this study.

The effects on changes in intestinal absorption in relation to GLP-2 treatment were evaluated in balance studies. Significant, clinically relevant and reproducible effects could be demonstrated on intestinal wet weight excretion and absorption over the two years of GLP-2 treatment. GLP-2, $400 \mathrm{mcg} / \mathrm{d}$ TID (corresponding to $0.018 \pm 0.002 \mathrm{mg} / \mathrm{kg} / \mathrm{d}$, range $0.014-0.020 \mathrm{mg} / \mathrm{d}$ ), reduced the fecal wet weight excretion from baseline values of approximately 3.0 to approximately $2.0 \mathrm{~kg} / \mathrm{d}$ after the first year of GLP- 2 treatment a reduction of the same magnitude was demonstrated during the second year. This reduction in the fecal wet weight excretion exceed the effects described in the initial 35 days GLP-2 study ( $400 \mathrm{mcg}$ BID corresponding to $0.013 \pm 0.002$ $\mathrm{mg} / \mathrm{kg} / \mathrm{d}$, range $0.011-0.017 \mathrm{mg} / \mathrm{kg} / \mathrm{d}$ ), where the fecal wet weight decreased from $3.2 \mathrm{~kg} / \mathrm{d}$ to $2.8 \mathrm{~kg} / \mathrm{d}$, and in the 21 days teduglutide study (doses ranging from 0.03 to $0.15 \mathrm{mg} / \mathrm{kg} / \mathrm{d}$ OD), where fecal wet weight decreased from 2.8 to $2.1 \mathrm{~kg} / \mathrm{d}$. However, in these studies the oral intakes were maintained constant as a part of the protocol.

In this study, the fecal wet weight excretion was not reduced by doubling the GLP-2 dose in six patients in this study for 3 weeks. Thus, the maximal effect may have been reached by the TID injection regimen.

The decrease in fecal wet weight excretion was gradually accompanied by a decline in the oral wet weight intake, 
thereby maintaining the intestinal wet weight absorption and urinary weight constant. In this study, parenteral support was kept constant during the entire study unless otherwise indicated by the patient and the investigator, and it was deliberately kept constant during the balance periods. An alternative approach would have been to taper the parenteral support according to the urinary production keeping the hyperphagic intake constant.

Since, the oral intake did not increase, and since the parenteral supplement of fluid and electrolytes was kept constant, the significant increases in urinary sodium, potassium and magnesium and the borderline significant increase in the urinary calcium excretion reflect the beneficial effects of GLP-2 on the intestinal absorption of these electrolytes. Although urinary volume did not increase significantly in relation to GLP-2 treatment, the renal function, evaluated by the renal creatinine clearance, improved significantly in this study. Renal impairment is a documented complication in HPN patients and is at least partially due to a relative chronic dehydration [39]. Since parenteral support is frequently administered during nighttime, many short bowel patients experience relative over-hydration during nighttime and relative dehydration during daytime. We speculate, that GLP2 treatment reduces the amplitude of the daily fluctuations in the fluid-balance of the short bowel patients, thereby reducing the daily interval, when dehydration is present. The positive effects on renal function are also reflected in the decrease in plasma-creatinine and the increase in plasma$\mathrm{CO}_{2}$-total in relation to GLP-2 treatment.

Although, the fecal energy excretion tended to decrease in relation to long-term GLP-2 treatment, this did not affect the overall intestinal energy absorption, since the dietary energy intake tended to decrease accordingly. In the shortterm studies employing native GLP-2 and teduglutide, the dietary intakes were fixed, and this may explain the positive trend towards an increase in the overall energy absorption. In the short-term study with native GLP-2, the absolute energy absorption tended to increase by approximately $400 \pm$ $600 \mathrm{~kJ} / \mathrm{d}(\sim 100 \mathrm{kcal} / \mathrm{d} ; P=.09)$. In the study using the dipeptidyl peptidase IV-resistant GLP-2 analog teduglutide, in doses 0.03 to $0.15 \mathrm{mg} / \mathrm{kg} / \mathrm{d}$, in 16 short-bowel patients (six with remnant parts of the colon), fecal energy excretion was reduced by approximately $800 \mathrm{~kJ} / \mathrm{d}(P=.04)$, but this only translated into a significant increase in intestinal absorption of approximately $1000 \mathrm{~kJ} / \mathrm{d}$ in a post hoc defined subset of patients with high dietary compliance during balance studies. In none of these three studies significant changes in the absorption of individual macronutrients were demonstrated. Although, a type-2 error is likely, the combination of GLP-2 and cholylsarcosine only tended to increase the fat absorption $(36 \pm 35 \mathrm{~g} / \mathrm{d}$ versus $59 \pm 26 \mathrm{~g} / \mathrm{d}$, $P=.08)$. However, all the patients receiving 2 grams of cholylsarcisine TID (8 capsules TID) responded, that they would not be able to continue this treatment long-term, and the clinical applicability therefore seems limited.

Originally, we believed that the marginal effects on intestinal energy absorption in the initial 35-days native GLP-2 study could explain the rather dramatic changes seen in body weight and body composition [15]. In the light of the negative findings on intestinal energy absorption, body weight and body composition in this long-term GLP-2 study, the increases described in body weight, lean body mass and the concurrent reduction in fat mass in the initial native GLP2 study could simply reflect a transient fluid retention in the initial phase of GLP-2 treatment prior to an adjustment to the new equilibrium.

In contrast to the initial native GLP-2 study, no changes were observed regarding bone mineral content in relation to long-term GLP-2 treatment. Again, acute fluctuations in calcium absorption or changes in bone formation and resorption could account for these conflicting findings. The GLP-2 dose currently suggested in the treatment of osteoporosis is $1600 \mu \mathrm{g}$ given at bedtime. Thus, the lower dose and the timing of injections may also explain the lack of effect of GLP-2 on bone mineral density in this study. In the initial study by Haderslev et al., positive effects on urinary excretions of markers of bone resorption, deoxypyridinoline and pyridioline, were described [17]. However, to compensate for day-to-day variation in urinary volume, these data were corrected for urinary creatinine excretion on the assumption that for each patient the 24hour urinary creatinine excretion was constant. However, since GLP-2 seems to affect urinary creatinine excretion by other means, the interpretation of these results may be incorrect. Thus, the clinical role of GLP-2 in the treatment of osteoporosis remains uncertain. It is currently investigated in postmenopausal women in a long-term, double blind, placebocontrolled trial, and studies on the physiological basis of the suggested effect of GLP-2 on bone resorption and formation are ongoing.

Since GLP-2 was initially believed to improve nutritional status in short bowel patients, we included measures of physical function in this study, including ${ }^{31} \mathrm{P}$ NMRS testing maximal aerobic ATP turn-over of red and white skeletal muscle. In accordance with the negative findings on body composition, none of these measures improved in relation to long-term GLP-2 treatment.

In conclusion, the main effect of GLP-2 treatment in patients with short bowel syndrome, where the parenteral support is kept constant, is a reduction in fecal losses of fluid and electrolytes. The effects on energy and macronutrient absorption are minor. This enables the patients to maintain their intestinal fluid and electrolyte absorption at lower oral intakes. A reduction in the amplitude in the fluctuations of the daily fluid-balance in relation to GLP-2 treatment may explain the beneficial effects on renal function.

An alternative approach to benefit from the positive effects of GLP-2 on intestinal absorption is to wean the SBS patients from parenteral support by encouraging them to maintain a hyperphagic oral intake during GLP-2 treatment. If the need for parenteral fluid support could be reduced by the magnitude of the reduction in fecal wet weight excretions, that is, approximately $1000 \mathrm{~g} / \mathrm{d}$, theoretically approximately $10-15 \%$ of short bowel patients with intestinal failure (based on evaluations of the HPN volumes needed in the Danish HPN-cohort) would be able to regain intestinal autonomy, and be weaned from HPN. They could have their central line removed in relation to treatment with GLP-2. 
It must, however, be emphasized, that all current data is obtained in open, nonrandomized studies and should be regarded as preliminary. However, the weaning strategy is currently tested in double blind, placebo-controlled trial. Although the preliminary results are inconsistent, it is likely that GLP-2 or analogs eventually could contribute to the limited treatments armamentarium of the short bowel syndrome.

\section{Abbreviations}

BC: Body Composition

BMD: Bone Mineral Density

DEXA: Dual-Energy X-ray Absorptiometry

GLP-2: Glucagon-like peptide 2

HPN: Home Parenteral Nutrition

SBS: Short Bowel Syndrome

NMR: Nuclear Magnetic Resonance

PTH: Parathyroid Hormone

\section{Acknowledgment}

The technical assistance of Jette Christiansen and Dorte Christensen (Department of Gastroenterology, Rigshospitalet, Denmark) as well as Hardi Hansen and Jan Borg Rasmussen (Department of Biomedical Sciences, Rigshospitalet) is greatly appreciated.

\section{References}

[1] C. Orskov, J. J. Holst, S. Knuhtsen, F. G. Baldissera, S. S. Poulsen, and O. V. Nielsen, "Glucagon-like peptides GLP1 and GLP-2, predicted products of the glucagon gene, are secreted separately from pig small intestine but not pancreas," Endocrinology, vol. 119, no. 4, pp. 1467-1475, 1986.

[2] M. Wojdemann, A. Wettergren, B. Hartmann, L. Hilsted, and J. J. Holst, "Inhibition of sham feeding-stimulated human gastric acid secretion by glucagon-like peptide-2," Journal of Clinical Endocrinology and Metabolism, vol. 84, no. 7, pp. 2513-2517, 1999.

[3] A. Bozkurt, E. Naslund, J. J. Holst, and P. M. Hellstrom, "GLP1 and GLP-2 act in concert to inhibit fasted, but not fed, small bowel motility in the rat," Regulatory Peptides, vol. 107, no. 13, pp. 129-135, 2002.

[4] M. Wojdemann, A. Wettergren, B. Hartmann, and J. J. Holst, "Glucagon-like peptide-2 inhibits centrally induced antral motility in pigs," Scandinavian Journal of Gastroenterology, vol. 33, pp. 828-832, 1998.

[5] X. Guan, B. Stoll, X. Lu, et al., "GLP-2-mediated up-regulation of intestinal blood flow and glucose uptake is nitric oxidedependent in TPN-fed piglets," Gastroenterology, vol. 125, no. 1, pp. 136-147, 2003.

[6] D. J. Drucker, P. Ehrlich, S. L. Asa, and P. L. Brubaker, "Induction of intestinal epithelial proliferation by glucagonlike peptide 2," Proceedings of the National Academy of Sciences of the United States of America, vol. 93, no. 15, pp. 7911-7916, 1996.

[7] D. J. Drucker, L. DeForest, and P. L. Brubaker, "Intestinal response to growth factors administered alone or in combination with human [Gly2] glucagon-like peptide 2," American Journal of Physiology, vol. 273, no. 6, pp. G1252-G1262, 1997.
[8] D. A. Litvak, M. R. Hellmich, B. M. Evers, N. A. Banker, and C. M. Townsend Jr., "Glucagon-like peptide 2 is a potent growth factor for small intestine and colon," Journal of Gastrointestinal Surgery, vol. 2, no. 2, pp. 146-150, 1998.

[9] C. Orskov, B. Hartmann, S. S. Poulsen, J. Thulesen, K. J. Hare, and J. J. Holst, "GLP-2 stimulates colonic growth via KGF, released by subepithelial myofibroblasts with GLP-2 receptors," Regulatory Peptides, vol. 124, no. 1-3, pp. 105-112, 2005.

[10] P. L. Brubaker, A. Izzo, M. Hill, and D. J. Drucker, "Intestinal function in mice with small bowel growth induced by glucagon-like peptide-2," American Journal of Physiology, vol. 272, no. 6, pp. E1050-E1058, 1997.

[11] C. I. Cheeseman and D. O’Neill, “Basolateral D-glucose transport activity along the crypt-villus axis in rat jejunum and upregulation induced by gastric inhibitory peptide and glucagon-like peptide-2," Experimental Physiology, vol. 83, no. 5, pp. 605-616, 1998.

[12] C. I. Cheeseman, "Upregulation of SGLT-1 transport activity in rat jejunum induced by GLP- 2 infusion in vivo," American Journal of Physiology, vol. 273, no. 6, pp. R1965-R1971, 1997.

[13] D. B. Henriksen, P. Alexandersen, B. Hartmann, et al., "Disassociation of bone resorption and formation by GLP-2. A 14-day study in healthy postmenopausal women," Bone, vol. 40, no. 3, pp. 723-729, 2007.

[14] L. Pironi, C. Zolezzi, E. Ruggeri, F. Paganelli, A. Pizzoferrato, and M. Miglioli, "Bone turnover in short-term and long-term home parenteral nutrition for benign disease," Nutrition, vol. 16, no. 4, pp. 272-277, 2000.

[15] P. B. Jeppesen, B. Hartmann, J. Thulesen, et al., "Glucagon-like peptide 2 improves nutrient absorption and nutritional status in short-bowel patients with no colon," Gastroenterology, vol. 120, no. 4, pp. 806-815, 2001.

[16] P. B. Jeppesen, E. L. Sanguinetti, A. Buchman, et al., "Teduglutide (ALX-0600), a dipeptidyl peptidase IV resistant qlucagonlike peptide 2 analogue, improves intestinal function in short bowel syndrome patients," Gut, vol. 54, no. 9, pp. 1224-1231, 2005.

[17] K. V. Haderslev, P. B. Jeppesen, B. Hartmann, et al., "Shortterm administration of glucagon-like peptide-2. Effects on bone mineral density and markers of bone turnover in short-bowel patients with no colon," Scandinavian Journal of Gastroenterology, vol. 37, no. 4, pp. 392-398, 2002.

[18] A. Schmassmann, H. F. Fehr, J. Locher, et al., "Cholylsarcosine, a new bile acid analogue: metabolism and effect on biliary secretion in humans," Gastroenterology, vol. 104, no. 4, pp. 1171-1181, 1993.

[19] A. Schmassmann, M. A. Angellotti, H. T. Ton-Nu, et al., "Transport, metabolism, and effect of chronic feeding of cholylsarcosine, a conjugated bile acid resistant to deconjugation and dehydroxylation," Gastroenterology, vol. 98, no. 1, pp. 163-174, 1990.

[20] S. Heydorn, P. B. Jeppesen, and P. B. Mortensen, "Bile acid replacement therapy with cholylsarcosine for short-bowel syndrome," Scandinavian Journal of Gastroenterology, vol. 34, no. 8, pp. 818-823, 1999.

[21] P. B. Jeppesen and P. B. Mortensen, "Colonic digestion and absorption of energy from carbohydrates and medium-chain fat in small bowel failure," Journal of Parenteral and Enteral Nutrition, vol. 23, no. 5, supplement, pp. S101-S105, 1999.

[22] P. B. Jeppesen, M. Staun, L. Tjellesen, and P. B. Mortensen, "Effect of intravenous ranitidine and omeprazole on intestinal absorption of water, sodium, and macronutrients in patients with intestinal resection," Gut, vol. 43, no. 6, pp. 763-769, 1998. 
[23] C. Rosenquist, C. Fledelius, S. Christgau, et al., "Serum CrossLaps One Step ELISA. First application of monoclonal antibodies for measurement in serum of bone-related degradation products from C-terminal telopeptides of type I collagen," Clinical Chemistry, vol. 44, no. 11, pp. 2281-2289, 1998.

[24] J. E. Cotes, D. J. Chinn, P. H. Quanjer, J. Roca, and J. C. Yernault, "Standardization of the measurement of transfer factor (diffusing capacity). Report Working Party Standardization of Lung Function Tests, European Community for Steel and Coal. Official Statement of the European Respiratory Society," The European Respiratory Journal, vol. 16, pp. 41-52, 1993.

[25] P. H. Quanjer, G. J. Tammeling, J. E. Cotes, O. F. Pedersen, R. Peslin, and J. C. Yernault, "Lung volumes and forced ventilatory flows. Report Working Party Standardization of Lung Function Tests, European Community for Steel and Coal. Official Statement of the European Respiratory Society," The European Respiratory Journal, vol. 16, pp. 5-40, 1993.

[26] L. F. Black and R. E. Hyatt, "Maximal respiratory pressures: normal values and relationship to age and sex," American Review of Respiratory Disease, vol. 99, no. 5, pp. 696-702, 1969.

[27] A. Ratkevicius and B. Quistorff, "Metabolic costs of force generation for constant-frequency and catchlike-inducing electrical stimulation in human tibialis anterior muscle," Muscle and Nerve, vol. 25, no. 3, pp. 419-426, 2002.

[28] L. Johansen and B. Quistorff, "31P-MRS characterization of sprint and endurance trained athletes," International Journal of Sports Medicine, vol. 24, no. 3, pp. 183-189, 2003.

[29] Y. Nakagawa, A. Ratkevicius, M. Mizuno, and B. Quistorff, "ATP economy of force maintenance in human tibialis anterior muscle," Medicine and Science in Sports and Exercise, vol. 37, no. 6, pp. 937-943, 2005.

[30] H. Galbo, M. Kjaer, and N. H. Secher, "Cardiovascular, ventilatory and catecholamine responses to maximal dynamic exercise in partially curarized man," Journal of Physiology, vol. 389, pp. 557-568, 1987.

[31] L. W. J. Bogert and J. J. van Lieshout, "Non-invasive pulsatile arterial pressure and stroke volume changes from the human finger," Experimental Physiology, vol. 90, no. 4, pp. 437-446, 2005.

[32] P. B. Jeppesen, P. Lund, I. B. Gottschalck, et al., "Short bowel patients treated for two years with Glucagon-Like Peptide 2 (GLP-2): compliance, safety and effects on quality of life," Gastroenterology Research and Practice, vol. 2009, 2009.

[33] M. Mizuno, A. Horn, N. H. Secher, and B. Quistorff, "Exerciseinduced 31P-NMR metabolic response of human wrist flexor muscles during partial neuromuscular blockade," American Journal of Physiology, vol. 267, no. 2, part 2, pp. R408-R414, 1994.

[34] H. I. Friedman, J. G. Chandler, C. C. Peck, T. J. Nemeth, and S. K. Odum, "Alterations in intestinal structure, fat absorption and body weight after intestinal bypass for morbid obesity," Surgery Gynecology and Obstetrics, vol. 146, no. 5, pp. 757-767, 1978.

[35] M. H. Gleeson, S. R. Bloom, J. M. Polak, K. Henry, and R. H. Dowling, "Endocrine tumour in kidney affecting small bowel structure, motility, and absorptive function," Gut, vol. 12, no. 10, pp. 773-782, 1971.

[36] F. M. Stevens, R. W. Flanagan, D. O'Gorman, and K. D. Buchanan, "Glucagonoma syndrome demonstrating giant duodenal villi,” Gut, vol. 25, no. 7, pp. 784-791, 1984.

[37] J. Thulesen, B. Hartmann, K. J. Hare, et al., "Glucagon-like peptide 2 (GLP-2) accelerates the growth of colonic neoplasms in mice," Gut, vol. 53, no. 8, pp. 1145-1150, 2004.
[38] A. Sylvan, B. Sjolund, K. G. Janunger, J. Rutegard, R. Stenling, and G. Roos, "Colorectal cancer risk after jejunoileal bypass: dysplasia and DNA content in longtime follow-up of patients operated on for morbid obesity," Diseases of the Colon and Rectum, vol. 35, no. 3, pp. 245-248, 1992.

[39] M. Lauverjat, A. H. Aissa, P. Vanhems, P. Bouletreau, D. Fouque, and C. Chambrier, "Chronic dehydration may impair renal function in patients with chronic intestinal failure on long-term parenteral nutrition," Clinical Nutrition, vol. 25, no. 1, pp. 75-81, 2006. 


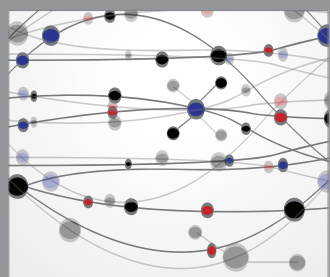

The Scientific World Journal
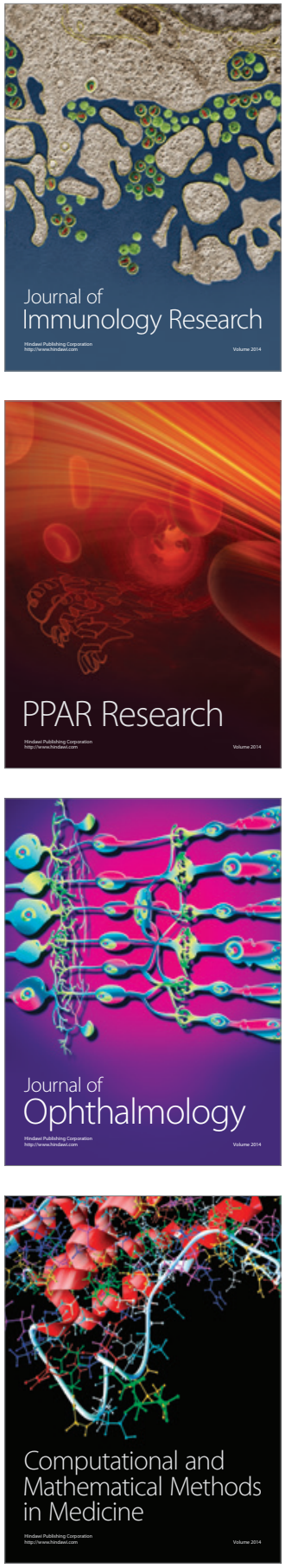

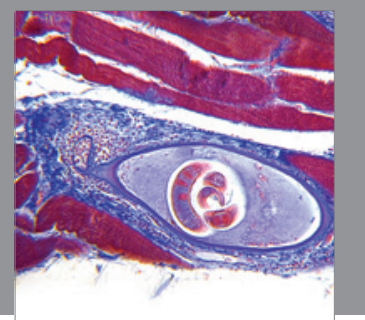

Gastroenterology

Research and Practice
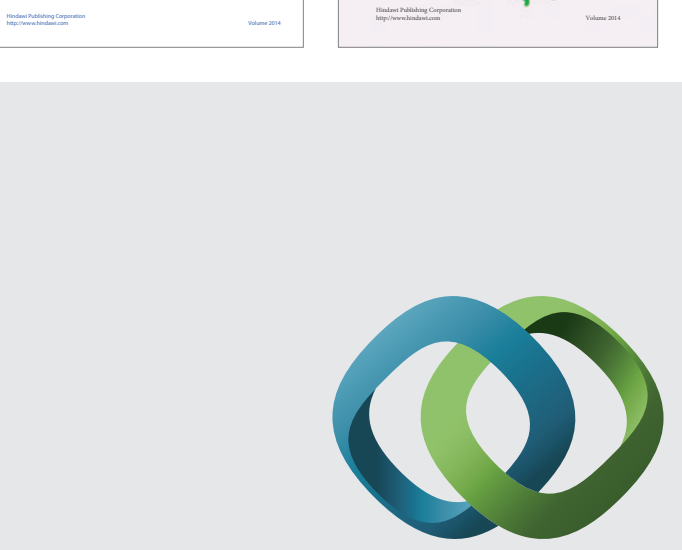

\section{Hindawi}

Submit your manuscripts at

http://www.hindawi.com
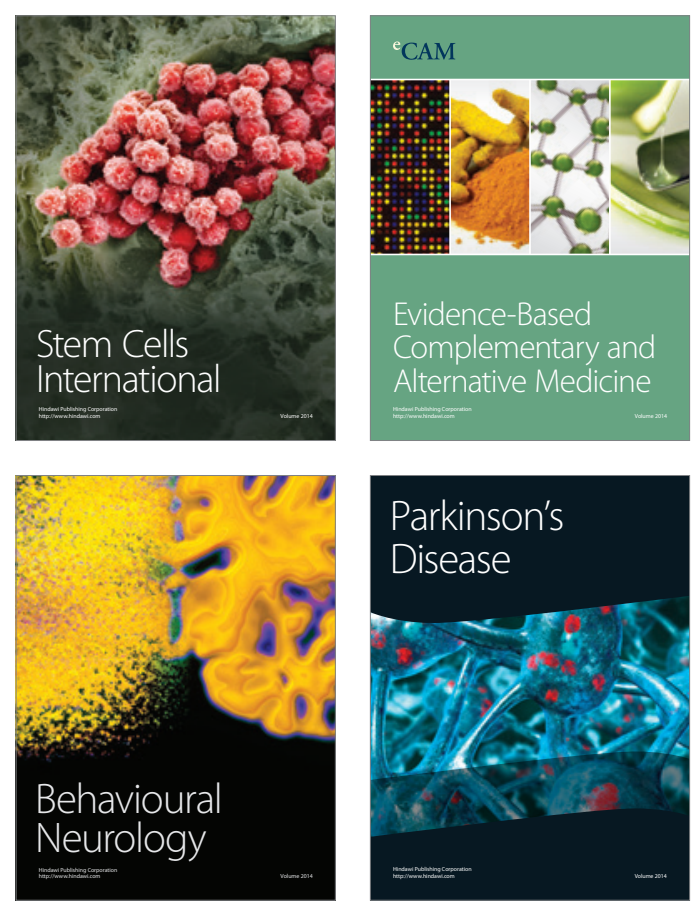

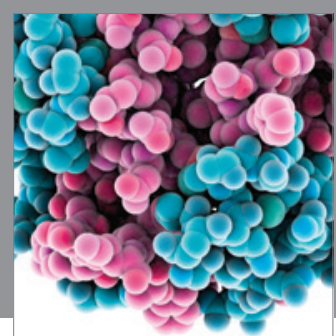

Journal of
Diabetes Research

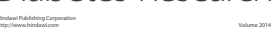

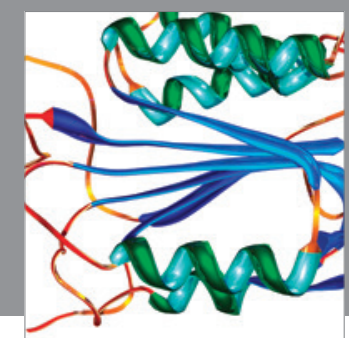

Disease Markers
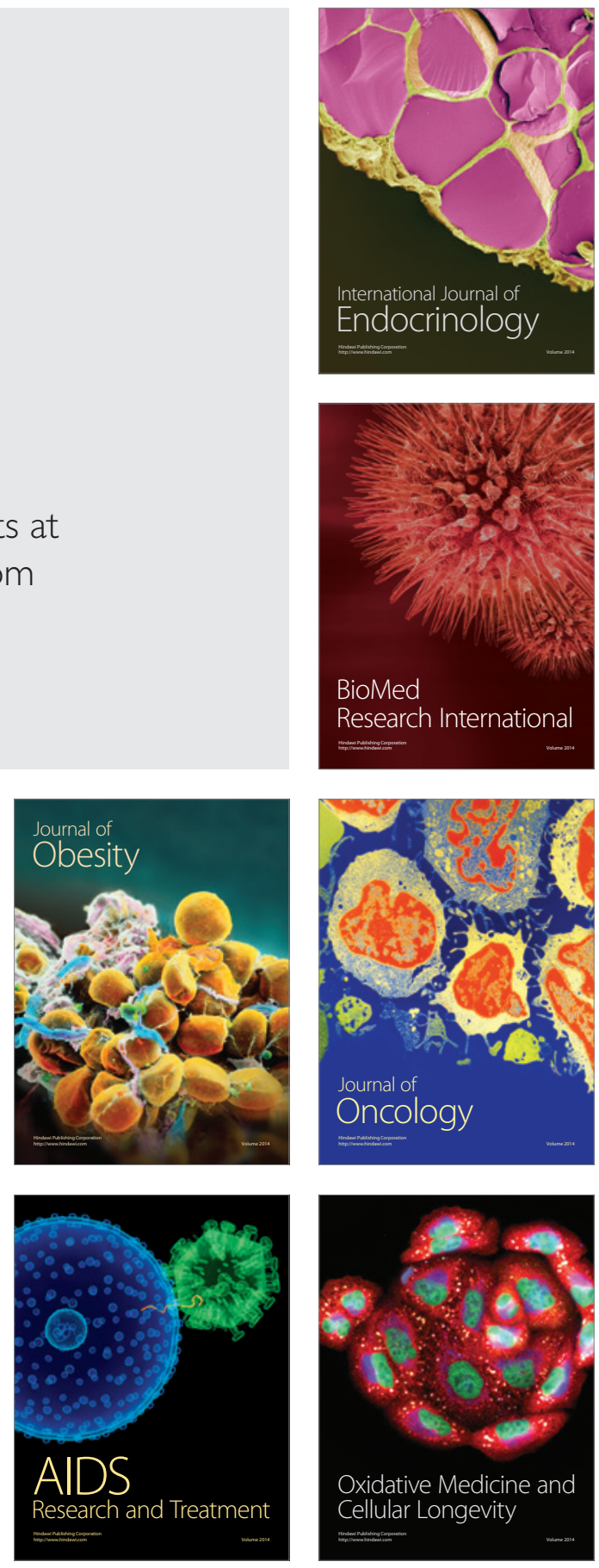